\title{
Isolation of Coronavirus NL63 from Blood from Children in Rural Haiti: Phylogenetic Similarities with Recent Isolates from Malaysia
}

\author{
Valery Madsen Beau De Rochars, ${ }^{1,2}$ John Lednicky, ${ }^{1,3}$ Sarah White, ${ }^{1,3}$ Julia Loeb,,$^{1,3}$ Maha A. Elbadry, ${ }^{1,3}$ Taina Telisma, ${ }^{1,4}$ \\ Sonese Chavannes, ${ }^{1,4}$ Marie Gina Anilis, ${ }^{1,4}$ Eleonora Cella, ${ }^{1,5,6}$ Massimo Ciccozzi, ${ }^{6}$ Bernard A. Okech, ${ }^{1,3}$ \\ Marco Salemi, ${ }^{1,5}$ and J. Glenn Morris Jr. ${ }^{1,7 *}$ \\ ${ }^{1}$ Emerging Pathogens Institute, University of Florida, Gainesville, Florida; ${ }^{2}$ Department of Health Services Research, Management and Policy, \\ College of Public Health and Health Professions, University of Florida, Gainesville, Florida; ${ }^{3}$ Department of Environmental and Global Health, \\ College of Public Health and Health Professions, University of Florida, Gainesville, Florida; ${ }^{4}$ Christianville Foundation, School Clinic, Gressier, \\ Haiti; ${ }^{5}$ Department of Pathology, Immunology and Laboratory Sciences, College of Medicine, University of Florida, Gainesville, Florida; \\ ${ }^{6}$ Department of Infectious Parasitic and Immunomediated Diseases, Reference Centre on Phylogeny, Molecular Epidemiology and Microbial \\ Evolution (FEMEM)/Epidemiology Unit, Istituto Superiore di Sanita, Rome, Italy; ${ }^{7}$ Department of Medicine, College of Medicine,
} University of Florida, Gainesville, Florida

\begin{abstract}
Human coronavirus (HCoV) NL63 is recognized as a common cause of upper respiratory infections and influenza-like illness. In screening children with acute undifferentiated febrile illness in a school cohort in rural Haiti, we identified HCoV-NL63 in blood samples from four children. Cases clustered over an 11-day period; children did not have respiratory symptoms, but two had gastrointestinal complaints. On phylogenetic analysis, the Haitian HCoV-NL63 strains cluster together in a highly supported monophyletic clade linked most closely with recently reported strains from Malaysia; two respiratory HCoV-NL63 strains identified in north Florida in the same general period form a separate clade, albeit again with close linkages with the Malaysian strains. Our data highlight the variety of presentations that may be seen with HCoV-NL63, and underscore the apparent ease with which CoV strains move among countries, with our data consistent with recurrent introduction of strains into the Caribbean (Haiti and Florida) from Asia.
\end{abstract}

Human coronaviruses (HCoVs) are being recognized with increasing frequency globally as a cause of human respiratory infections. ${ }^{1-8}$ Strains are also associated with infections in animals, with current data suggesting that bats are a primary reservoir and point of origin for HCoVs. ${ }^{9,10}$ Initially described in the 1960s, interest in the coronaviruses increased substantially in the early 2000 with the emergence of severe acute respiratory syndrome (SARS), ${ }^{11}$ followed by the Middle East respiratory syndrome (MERS) coronaviruses (CoV) in 2012. ${ }^{12}$ Other than SARS-CoV and MERS-CoV, which have tended to occur in more delimited epidemics, four coronaviruses are currently recognized as being endemic in human populations: HCoV-229E, HCoV-OC43, HCoVNL63, and HCoV-HKU1. ${ }^{1-8}$

Our group works closely with the school clinic that serves four schools operated by the Christianville Foundation in the Gressier/Leogane region of Haiti, some 20 miles west of Port-au-Prince. The schools have a total of approximately 1,250 students, from prekindergarten to grade $12 .{ }^{13}$ Because of our interest in monitoring arboviral transmission within this population, we have protocols in place for the collection of diagnostic blood samples from children presenting to the school clinic with acute undifferentiated febrile illness (i.e., febrile illness with no localizing signs, such as would be expected with pneumonia, upper respiratory infections, urinary tract infections). The protocol for sample collection was approved by the University of Florida Institutional Review Board (IRB) and the Haitian National IRB, and written parental informed consent was obtained from parents or guardians of all study participants.

*Address correspondence to J. Glenn Morris Jr., Emerging Pathogens Institute, University of Florida, 2055 Mowry Road, P.O. Box 100009, Gainesville, FL 32610-0009. E-mail: jgmorris@epi.ufl.edu

\section{CASE REPORTS}

Between May 2014 and February 2015, blood samples were obtained at the school clinic from a total of 177 children who met criteria for the diagnosis of acute undifferentiated febrile illness. HCoV-NL63 was identified in samples from four case patients seen between January 16 and January 27, 2015. Ages ranged from 3 to 10 years (mean 6 years). All presented with subjective symptoms of fever, although only two were febrile when examined $\left(39^{\circ} \mathrm{C}\right.$ and $38.5^{\circ} \mathrm{C}$, respectively). Two complained of headache, whereas two complained of "abdominal flu." In keeping with the criteria for enrollment in the study, no respiratory symptoms or symptoms consistent with croup were reported. Routine stool cultures and examination for ova and parasites were performed on stool samples from all four children, as previously described ${ }^{13}$ : Giardia was identified in the stool sample of one of the children with abdominal complaints, and Blastocystis was identified in one of the children with no gastrointestinal (Gl) complaints. No bacterial pathogens were identified on stool culture. Malaria screens on all four children were negative. In all instances, illness was mild and self-limited, and children recovered without sequelae.

Plasma samples were cultured using cell lines and conditions as previously described, ${ }^{14,15}$ and as reported in detail in the Supplemental Information that accompanies this publication. In brief, aliquots of plasma $(25-100 \mu \mathrm{L})$ from each patient were inoculated in duplicate onto five different cell lines (A549, LLC-MK2, MDCK, MRC5, and Vero E6). Cell cultures from each of the four patients displayed cytopathic effects (CPEs) that were clearly different from those observed in cells in which alpha-, flavi-, and other viruses were isolated (Figure 1). The CPE were most obvious in LLC-MK2 cells and less so in Vero E6 incubated at either $33^{\circ} \mathrm{C}$ or $37^{\circ} \mathrm{C}$.

Primary screens of spent culture from these cell cultures by reverse transcription polymerase chain reaction (RT-PCR) 

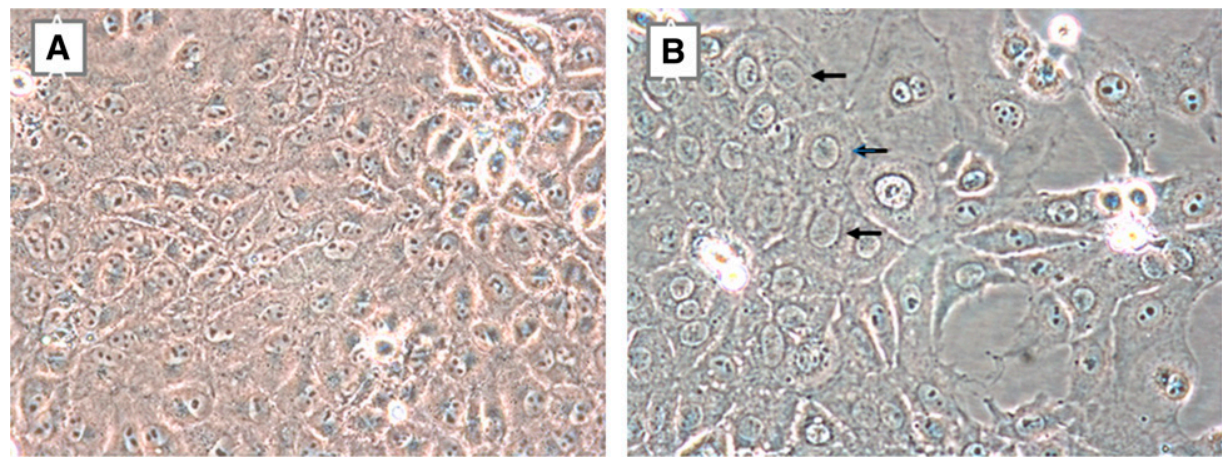

FIGURE 1. LLC-MK2 cells. Noninoculated cells (A) appear crowded 13 days postseed; no spaces are present between the cells and the nuclei have prominent nucleoli. (B) Cells 13 days postinoculation with plasma sample four appear different: the nuclei of the infected cells appear larger, lack prominent nucleoli, and have visibly darker nuclear borders (black arrows), and clearings due to detachment of dead cells are evident, as are a few refractile floating dead cells. The virus grew at both $33^{\circ} \mathrm{C}$ and $37^{\circ} \mathrm{C}$; this figure shows virus in cells grown at $33^{\circ} \mathrm{C}$. The appearance of the nuclei of the infected LLC-MK2 cells is consistent with HCoV-NL63 infections. Localization to the nucleolus is a common feature of coronavirus nucleoproteins, resulting in the accumulation of cells in the M-phase of the cell cycle (nucleoli are absent in dividing cells), and the formation in syncytia in a fraction of the cells. Moreover, immature HCoV-NL63 particles form in the rough endoplasmic reticulum (RER) surrounding the nucleus, and the dark border surrounding the enlarged nuclei in Figure 1B are likely due to cellular changes in the RER of the infected cells. Representative photomicrographs of cytopathic effects of other viral species are shown in Supplemental Figures 1-3.

were negative for arena-, alpha-, entero-, and flavivirus viral genomic RNAs (vRNAs); however, they were positive for HCoV-NL63 using the GenMark eSensor XT-8 RVP system (eSensor RVP; GenMark Diagnostics, Inc., Carlsbad, CA). The agent isolated in these cultures was subsequently confirmed as HCoV-NL63 by RT-PCR and sequencing of the amplicons and the virus genome. Similar methods were used to obtain sequence data for HCoV-NL63 from two positive respiratory samples collected in July and September 2015, respectively, at University of Florida Health/ Shands Hospital in Gainesville, FL. GenBank accession numbers: HCoV-NL63/Haiti: KT266906; KX179494- KX179499. HCoV-NL63/Florida: KT381875.1; KU521535.1.

A multiple sequence alignment was assembled including 192 HCoV-NL63 spike gene region reference sequences, downloaded from National Center for Biotechnology Information (see Supplemental Table 1 for details about the reference sequences), plus the four Haitian sequences and the two sequences from University of Florida Health. The locations of the reference sequences considered for the analysis were Belgium, China, Ghana, Hong Kong, Japan, Malaysia, Netherlands, Sweden, Thailand, and the United States. The evolutionary model was chosen as the best-fitting nucleotide substitution model in accordance with the results of the hierarchical likelihood ratio test implemented with the Modeltest software version 3.7. ${ }^{16}$ See supplemental Information for a complete description of phylogenetic methods.

Bayesian factor (BF) analysis showed that a significant better fit for the relaxed rather than the strict molecular clock model (LnBF > 15 in favor of the former). Under the relaxed clock, the BF analysis also showed that the nonparametric Bayesian skyline plot was the best-fitting demographic model (LnBF > 53 for each comparison). The estimated mean evolutionary rate was $7.02 \times 10^{-4}$ substitutions/site/year (95\% high posterior density [HPD] $3.97 \times 10^{-4}-1.09 \times 10^{-3}$ ). The Maximum Clade Credibility tree shows three main supported clades (Figure 2) with no particular geographic structure, although it is clear that the older lineages, close to the root of the tree are from United States with a most recent common ancestor dating back to the mid-1970s (a tree with reference labels is provided in Supplemental Figure 4).

The four new Haitian isolates belonged to genotype $C$ and clustered within a well-supported $(P>0.95)$ sub-clade with a most recent common ancestor dating back to 2013 (95\% high posterior density intervals 2012-2014). The two Florida sequences also clustered with genotype $\mathrm{C}$ sequences and formed a separate but highly supported sub-clade $(P>$ 0.95), with a most recent common ancestor dating back to 2014 (95\% HPD intervals 2013.5-2014.5). The Haitian and Florida isolates and a strain from Malaysia are part of a larger sub-clade branching off independently from several genotype C Malaysian sequences, ${ }^{17}$ which would suggest an Asian origin for the new strains, although the sub-clade does not have strong statistical support $(P<0.9)$. Overall, the data are consistent with at least two recent and independent introductions of HCoV-NL63 virus in the region (one in Haiti, one in north Florida), possibly originating from east Asia.

\section{DISCUSSION}

HCoV-NL63 is widely distributed in human populations, and has been isolated from patients with symptoms ranging from those of a mild "common cold" to influenza-like illness, and, rarely, pneumonia and more severe lower respiratory infections. ${ }^{1-8}$ Cases tend to cluster, often in winter/late winter months in temperate areas, although there is a wide variation in seasonality in different parts of the world. In comparison to the other common HCoV groups, NL63 has been reported as the most common cause of croup in infants $<6$ months of age. ${ }^{7}$ It is also commonly seen as a coinfection with other known pathogenic viruses $(38 \%$ of total cases in one study were infected with two or more viruses ${ }^{7}$ ), and, in a study in Ghana, which compared children with respiratory symptoms with control children without such symptoms, HCoV-NL63 was significantly more 


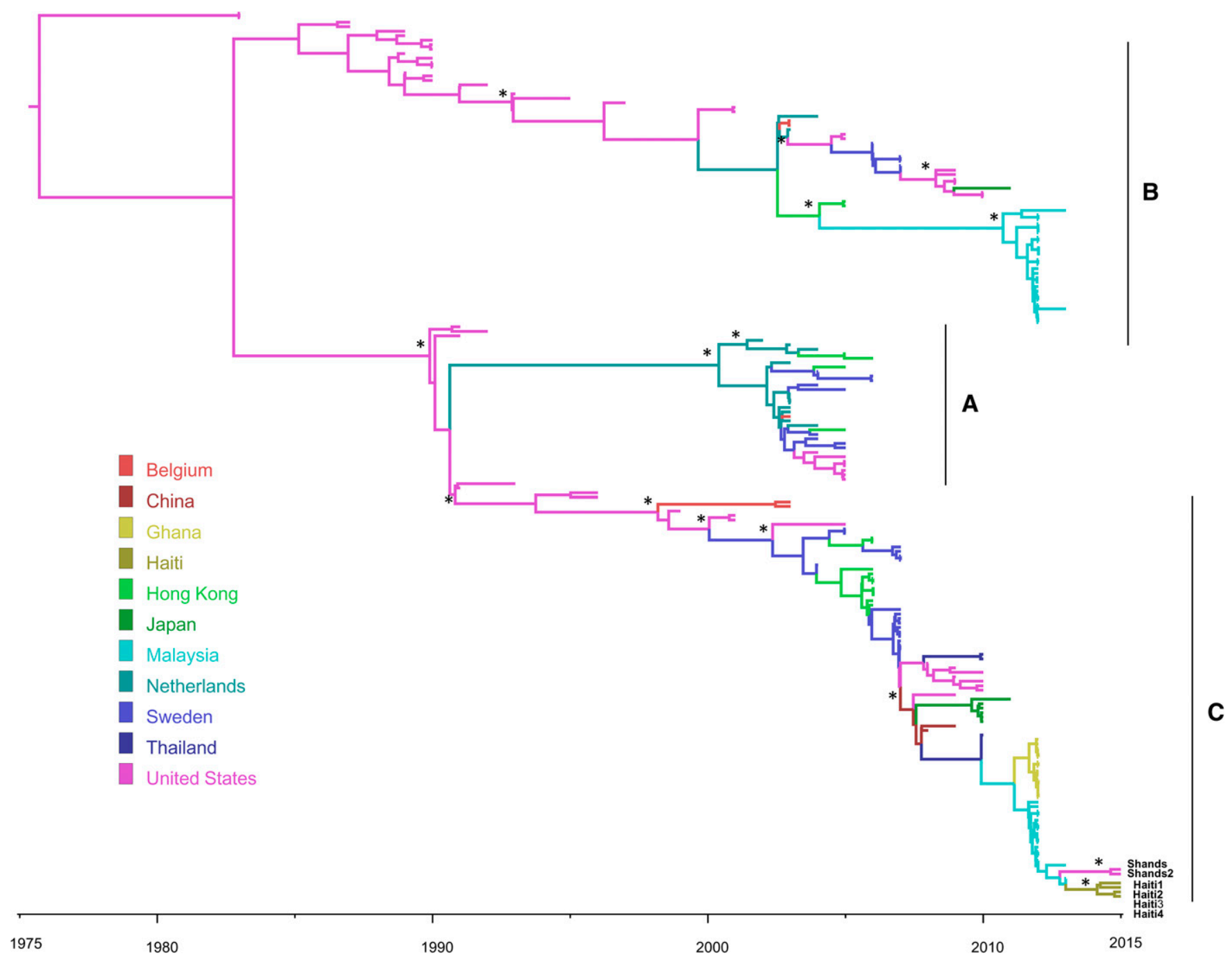

FIGURE 2. Maximum Clade Credibility tree of HCoV-NL63 spike gene region. Branches are colored according to the legend to the left where each color represents the geographic location of the sampled sequence (tip branches), as well as of the ancestral lineage (internal branches) inferred by Bayesian phylogeography. The tree was scaled in time by enforcing a relaxed molecular clock (see Supplemental Information). One asterisk along the branch represents significant statistical support (posterior probability $\geq 90 \%$ ). Sequence labels for the new strains are shown.

common among control children than case children $(8.5 \%$ versus $6.8 \%, P=0.022){ }^{3} \mathrm{HCoV}$ (including HCoV-NL63) have also been identified in stool samples from children with acute gastroenteritis; however, $82 \%$ of patients from whom HCoV were isolated also had either norovirus or rotavirus in the stool sample. ${ }^{18}$ Taken together, these data paint a picture of a widely distributed virus, present in the respiratory and GI tract, with uncertain pathogenicity, possibly due to inherently low virulence and/or the impact of high levels of prior immunity in the general population.

In this study, we isolated HCoV-NL63 from a small cluster of children with febrile illness, without respiratory symptoms, in January in rural Haiti. As our primary goal was to screen for arboviruses, we specifically excluded children with respiratory symptoms from sample collection, so we cannot be certain how many of this latter group might also have been infected with/carrying HCoV-NL63. What was unexpected was the isolation of this specific coronavirus group from blood, which, to our knowledge, has not been previously reported. However, viremia is not totally unexpected: HCoVNL63 is capable of infecting human kidney cells in tissue culture $^{19}$ by using the same cell receptor (ACE2 receptor) as SARS-CoV, which causes systemic illness, and is shed in respiratory secretions, urine, and stools, with presumptive hematogenous spread. The clinical significance of the viremia in our specific cases is uncertain. All case patients were febrile, and two had Gl complaints (although one of the two with Gl complaints also had Giardia on stool examination). Our findings indicate that viremia with HCoV-NL63 does occur, and raise the possibility that it can cause a mild febrile illness in otherwise healthy children, without respiratory manifestations, but, possibly, with diarrhea.

At a phylogenetic level, our Haitian isolates were most closely linked with isolates from Malaysia. We also sequenced two HCoV-NL63 isolates from respiratory samples at University of Florida Health in Gainesville: they clustered together within a separate monophyletic clade, with a separate link back to Malaysian strains. These findings underscore the 
apparent ease with which coronavirus strains can spread at a global level, findings in keeping with the observed continental jumps of both SARS-CoV and MERS-CoV. ${ }^{20}$

Received July 19, 2016. Accepted for publication September 16, 2016.

Published online October 31, 2016.

Note: Supplemental information, table, and figures appear at www. ajtmh.org.

Authors' addresses: Valery M. Beau de Rochars, Emerging Pathogens Institute, University of Florida, Gainesville, Florida and Department of Health Services Research, Management and Policy, University of Florida, Gainesville, FL, E-mail: madsenbeau@phhp.ufl.edu. John Lednicky, Sarah White, Julia Loeb, Maha A. Elbadry, and Bernard A. Okech, Emerging Pathogens Institute, University of Florida, Gainesville, FL and Department of Environmental and Global Health, College of Public Health and Health Professions, University of Florida, Gainesville, FL, E-mails: jlednicky@phhp.ufl.edu, sek0005@ ufl.edu, jloeb@phhp.ufl.edu, elbadrym@ufl.edu, and bokech@ufl.edu. Taina Telisma, Sonese Chavannes, and Marie Gina Anilis, Emerging Pathogens Institute, University of Florida, Gainesville, Florida and Christianville Foundation, School Clinic, Gressier, Haiti, E-mails: tainaquisqueya@gmail.com, chavannessonse@yahoo.fr, and mariegina anilis@yahoo.fr. Eleonora Cella, Emerging Pathogens Institute, University of Florida, Gainesville, FL, Department of Pathology, Immunology and Laboratory Sciences, College of Medicine, University of Florida, Gainesville, Florida, and Department of Infectious Parasitic and Immunomediated Diseases, Reference Centre on Phylogeny, Molecular Epidemiology and Microbial Evolution (FEMEM)/Epidemiology Unit, Instituto Superiore de Sanita, Rome, Italy, E-mail: eleonora. cella@yahoo.it. Massinno Ciccozzi, Department of Infectious Parasitic and Immunomediated Diseases, Reference Centre on Phylogeny, Molecular Epidemiology and Microbial Evolution (FEMEM)/ Epidemiology Unit, Istituto Superiore di Sanita, Rome, Lazio, Italy, E-mail: ciccozzi@iss.it. Marco Salemi, Emerging Pathogens Institute, University of Florida, Gainesville, FL, and Department of Pathology, Immunology and Laboratory Sciences, College of Medicine, University of Florida, Gainesville, FL, E-mail: salemi@pathology.ufl.edu. J. Glenn Morris Jr., Emerging Pathogens Institute, University of Florida, Gainesville, FL, and Department of Medicine, College of Medicine, University of Florida, Gainesville, FL, E-mail: jgmorris@epi.ufl.edu.

\section{REFERENCES}

1. Berry M, Gamieldien J, Felding BC, 2015. Identification of new respiratory viruses in the new millennium. Viruses 5: 996-1019.

2. Yip CCY, Lam CSF, Luk HKH, Wong EYM, Lee RA, So L-Y, Chan K-H, Cheng ACC, Yuen K-Y, Woo PCY, Lau SKP, 2016. A six-year descriptive epidemiological study of human coronavirus infections in hospitalized patients in Hong Kong. Virol Sin 31: 41-48.

3. Owusu M, Annan A, Corman VM, Larbi R, Anti P, Drexler JF, Agbenyega O, Adu-Sarkodie Y, Drosten C, 2014. Human coronavirus associated with upper respiratory tract infections in three rural areas of Ghana. PLoS One 9: e99782.

4. Matoba Y, Abiko C, Ikeda T, Aoki Y, Suzuki Y, Yahagi K, Matsuzaki Y, Itagaki T, Katsushima F, Katsuchima Y, Mizuta $\mathrm{K}, 2015$. Detection of human coronavirus 229E, HKU1, NL 63, and OC43. Jpn J Infect Dis 68: 138-141.

5. Huang S-H, Su M-C, Tien N, Huang C-J, Lan Y-C, Lin C-S, Chen C-H, Lin C-W, 2015. Epidemiology of human coronavirus NL63 infection among hospitalized patients with pneumonia in Taiwan. J Microbiol Immunol Infect. doi: 10.1016/ j.jmii.2015.10.008.

6. Razuri JH, Malecki M, Tinoco Y, Ortiz E, Guezala MC, Romero C, Estela A, Brena P, Morales M-L, Reaves EJ, Gomez J,
Uyeki TM, Widdowson M-A, Azziz-Baumgartner Bausach DG, Schildgen V, Schildgen O, MontgomeryJM, 2015. Human coronavirus-associated influenza-like illness in the community setting in Peru. Am J Trop Med Hyg 93: 1138-1140.

7. Lee J, Storch GA, 2014. Characterization of human coronavirus OC43 and human coronavirus NL63 infections among hospitalized children $<5$ years of age. Pediatr Infect Dis $J 33$ : 814-820.

8. Trombetta H, Faggion HZ, Leotte J, Nogueira MB, Vidal LRR, Raboni SM, 2016. Human coronavirus and severe acute respiratory infection in southern Brazil. Pathog Glob Health 110: $113-118$

9. Hu B, Ge X, Wang L-F, Shi Z, 2015. Bat origin of human coronaviruses. Virol J 12: 221

10. Huynh J, Li S, Yount B, Smith A, Sturges L, Olsen JC, Nagel J, Johnson JB, Agnihothram S, Gates JE, Frieman MB, Baric RS, Donaldson EF, 2012. Evidence supporting a zoonotic origin of human coronavirus strain NL63. J Virol 86: 12816-12825.

11. Ksiazek TG, Erdman D, Goldsmith CS, Zaki SR, Peret T, Emery S, Tong S, Urbani C, Comer JA, Lim W, Rollin PE, Dowell SF, Ling AE, Humphrey CD, Shieh WJ, Guarner J, Paddock CD, Rota P, Fields B, DeRisi J, Yang JY, Cox N, Hughes JM, LeDuc JW, Bellini WJ, Anderson LJ, SARS Working Group, 2003. A novel coronavirus associated with severe acute respiratory syndrome. N Engl J Med 348: 1953-1966.

12. de Groot RJ, Baker SC, Baric RS, Brown CS, Drosten C, Enjuances L, Rouchier RAM, Galiano M, Gorbalenya AE, Memish ZA, Perlman S, Poon LLM, Snijder EJ, Stephens GM, Woo PCY, Zaki AM, Zambon M, Ziebuhr J, 2013. Middle east respiratory syndrome coronavirus (MERS-CoV): announcement of the Coronavirus Study Group. J Virol 87: 7790-7792.

13. Beau De Rochars VEM, Alam MT, Telisma T, Masse R, Chavannes S, Anilis MG, Guillaume JH, Gelin G, Kirkpatrick EL, Desormeaux A-M, Okech BA, Weppelmann TA, Rashid M, Karst S, Johnson JA, Ali A, Morris JG Jr, 2015. Spectrum of outpatient illness in a school-based cohort in Haiti, with a focus on diarrheal pathogens. Am J Trop Med Hyg 92: 752-757.

14. Lednicky J, Beau de Rochars VM, ElBadry M, Loeb J, Telisma T, Chavannes S, Anilis G, Cella E, Ciccozzi M, Rashid M, Okech B, Salemi M, Morris JG Jr, 2016. Zika virus outbreak in Haiti in 2014: molecular and clinical data. PLoS Negl Trop Dis 10: e0004687.

15. El Badry M, Lednicky J, Cella E, Telisma T, Chavannes S, Loeb J, Ciccozzi M, Okech B, Beau De Rochars VM, Salemi M, Morris JG Jr, 2016. Isolation of an entervirus D68 from blood of a child with pneumonia in rural Haiti: close phylogenetic linkage with New York strain. Ped Infect Dis J 35: $1048-1050$

16. Posada D, Buckley TR, 2004. Model selection and model averaging in phylogenetics: advantages of akaike information criterion and bayesian approaches over likelihood ratio tests. Syst Biol 53: 793-808.

17. Al-Khannaq MN, Ng KT, Oong XY, Pang YK, Takebe Y, Chook JB, Hanafi NS, Kamarulzaman A, Tee KK, 2016. Diversity and evolutionary histories of human coronaviruses NL63 and $229 \mathrm{E}$ associated with acute upper respiratory tract symptoms in Kuala Lumpur, Malaysia. Am J Trop Med Hyg 94: 1058-1064.

18. Risku M, Lappalainen S, Rasanen S, Vesikari T, 2010. Detection of human coronaviruses in children with acute gastroenteritis. $J$ Clin Virol 48: 27-30.

19. Lednicky JA, Waltzek TB, McGeehan E, Loeb JC, Hamilton SB, Luetke MC, 2013. Isolation and genetic characterization of human coronavirus NL63 in primary human renal proximal tubular epithelial cells obtained from a commercial supplier, and confirmation of its replication in two different types of human primary kidney cells. Virol J 10: 213.

20. Cowling BJ, Park M, Fang VJ, Wu P, Leung GM, Wu JT, 2015. Preliminary epidemiological assessment of MERS-CoV outbreak in South Korea, May to June 2015. Euro Surveill 20: 21163. 